\title{
Expression of Hcy and blood lipid levels in serum of CHD patients and analysis of risk factors for CHD
}

\author{
WEIJING LIU, TAO WANG, PENGFEI SUN and YANJU ZHOU \\ Department of Emergency, Weifang People's Hospital, Weifang, Shandong 261000, P.R. China
}

Received May 16, 2018; Accepted November 15, 2018

DOI: $10.3892 / \mathrm{etm} .2018 .7111$

\begin{abstract}
Expression of homocysteine (Hcy) and blood lipid levels in serum of atherosclerotic coronary heart disease (CHD) patients was investigated and the related risk factors were analyzed. A total of 148 CHD patients were selected as study group and another 120 healthy people attending Weifang People's Hospital for physical examination in the same period as control group. Hcy and blood lipid levels in serum were measured in the two groups, including total cholesterol (TC), triglyceride (TG), high-density lipoprotein cholesterol (HDL-C) and low-density lipoprotein cholesterol (LDL-C). At the same time, analysis of risk factors for CHD was performed. The levels of Hcy, TC, TG and LDL-C in patients in the study group were significantly higher than that in control group, while the HDL-C level was significantly lower than that in control group, and the differences were statistically significant $(\mathrm{P}<0.001)$. The difference was statistically significant in the levels of Hcy, TC, TG and LDL-C between acute and old myocardial infarction and angina pectoris group $(\mathrm{P}<0.05)$, and the difference was statistically significant in the levels of Hcy, TC, TG and LDL-C between old and acute myocardial infarction group $(\mathrm{P}<0.05)$. Analysis of risk factors for the disease was performed using logistic regression. The results of univariate analysis showed that CHD was associated with Hcy, hyperlipidemia, smoking, hypertension and diabetes $(\mathrm{P}<0.05)$, but had no relationship with drinking and obesity $(\mathrm{P}>0.05)$. The results of multivariate analysis showed that Hcy, hyperlipidemia, hypertension and diabetes were independent risk factors for $\mathrm{CHD}$, and the difference was statistically significant $(\mathrm{P}<0.05)$. The Hcy level increased and the HDL-C level decreased in serum of CHD patients. Hcy, hyperlipidemia, hypertension and diabetes are independent risk factors for CHD, which can provide practical basis for the diagnosis, treatment and prevention.
\end{abstract}

Correspondence to: Dr Yanju Zhou, Department of Emergency, Weifang People's Hospital, 151 Guangwen Street, Weifang, Shandong 261000, P.R. China

E-mail: hnrrry9003@163.com

Key words: homocysteine, blood lipid, coronary heart disease, risk factor

\section{Introduction}

The morbidity and mortality of atherosclerotic coronary heart disease (CHD) have increased with the development of economy and the improvement of human living standard, and heart disease is ranked first of the diseases seriously threatening human life and health at present (1). Studies have shown that male incidence is higher than female, it is not completely clear what the specific pathogenesis of CHD is, and there are various theories, including endothelial dysfunction, inflammatory reaction and lipid deposition hypothesis $(2,3)$. In addition to sex, age and blood lipid, the pathogenic factors affecting CHD are mainly related to blood sugar, blood pressure, obesity, smoking, and drinking. In recent years, a large number of studies have proved that homocysteine (Hcy) is also one of the risk factors for CHD (4-6).

Hcy, a non-essential amino acid, is converted mainly from methionine intake in the diet (7). It has been reported that Hcy may damage vascular endothelial cells and affect lipid metabolism, contributing to the occurrence of atherosclerosis, thus inducing cardiovascular and cerebrovascular diseases, but it is not completely clear what the mechanism is $(8,9)$. Abnormal lipid metabolism is the most important risk factor in atherosclerosis. Generally speaking, indicators of blood lipid include total cholesterol (TC), triglyceride (TG), high-density lipoprotein cholesterol (HDL-C) and low-density lipoprotein cholesterol (LDL-C) (10). Studies have found that Hcy participates in and speeds up the auto-oxidation of LDL, while oxidized LDL cannot be recognized by normal LDL receptors, leading to the occurrence and development of acute coronary syndrome (11).

The purpose of this study is to compare the Hcy and blood lipid level in serum of CHD patients with that of healthy people and to perform statistical analysis on the risk factors for CHD, to provide reference value for the assessment of disease and the prevention and prognosis of CHD.

\section{Patients and methods}

General information. A total of 148 patients who were diagnosed as CHD patients by coronary arteriography in Weifang People's Hospital (Weifang, China) between January 2014 and December 2017 were collected as study group using retrospective methods, including 89 male and 
59 female with an average age of $56.42 \pm 9.84$ years. The study group was divided into 67 cases in angina pectoris group, 49 cases in acute myocardial infarction group and 32 cases in old myocardial infarction group according to the clinical characteristics. Another 120 healthy people in the same period were selected as control group, including 64 males and 56 females with an average age of 54.21 \pm 12.0 years. Exclusion criteria were: Subjects who were pregnant, with lactation, tumors, liver and renal insufficiency and various infections and immune diseases. No drugs affecting Hcy metabolism were used and no steroid drug therapy was received by the patients within the 2 months of the study. A total of 268 subjects signed the informed consent. General information on the candidates is shown in Table I. The study was approved by the Ethics Committee of Weifang People's Hospital.

Instruments and reagents. The DNM-9606 enzyme marker analyzer was purchased from Beijing Plang New Technology Co., Ltd. (Beijing, China); Hcy ELISA kit from Xiamen Huijia Biotechnology Co., Ltd. (Xiamen, China); HI7100 biochemistry analyzer and its reagents from Hitachi, Ltd. (Tokyo, Japan); blood lipid kit from Shanghai Yubo Biotechnology Co., Ltd. (Shanghai, China).

Determination of Hcy and blood lipid levels in serum. All subjects were fasting for $>12 \mathrm{~h}$ on an empty stomach before examination and no smoking, drinking and high-fat food for $24 \mathrm{~h}$. Venous blood $(3 \mathrm{ml})$ was collected from patients on an empty stomach in the study and control group, and Hcy and blood lipid content in serum were determined using centrifugation at $8,000 \times \mathrm{g}$ for $20 \mathrm{~min}$ at $4^{\circ} \mathrm{C}$. The Hcy level in serum was determined quantitatively using enzyme-linked immunosorbent assay. TC was determined using enzyme method, TG using glycerol phosphate oxidase method and HDL-C and LDL-C using selective inhibition method. The indicator of each group was determined 3 times and its average value was taken. All steps were strictly in accordance with the instructions.

Statistical analysis. Data analysis was performed using SPSS 17.0 statistical software (SPSS, Inc., Chicago, IL, USA). The enumeration data were tested by $\chi^{2}$ test, the comparison of measurement data between groups was tested by t-test, the comparison among multiple groups was performed using one-way ANOVA, and pairwise comparison between groups was performed using SNK-q test. Analysis of risk factors for the disease was performed using logistic regression. At $\mathrm{P}<0.05$, the difference was considered statistically significant.

\section{Results}

Comparison of Hcy and blood lipid levels in serum between the two groups. Comparison of Hcy and blood lipid levels in serum between the study and control group was performed. The HDL-C level in the study group was significantly lower than that in control group, while the levels of Hcy, TC, TG and LDL-C were significantly higher than that in control group, and the differences were statistically significant $(\mathrm{P}<0.001)$. Specific information is shown in Fig. 1 and Table II.
Table I. General information [n (\%)].

\begin{tabular}{|c|c|c|c|c|}
\hline Factors & $\begin{array}{l}\text { Study group } \\
\quad(n=148)\end{array}$ & $\begin{array}{l}\text { Control group } \\
\quad(n=120)\end{array}$ & $\chi^{2}$ & P-value \\
\hline \multicolumn{5}{|l|}{ Age (years) } \\
\hline$\geq 54$ & $102(68.92)$ & $72(60.00)$ & 2.315 & 0.128 \\
\hline$<54$ & $46(31.08)$ & $48(40.00)$ & & \\
\hline \multicolumn{5}{|l|}{ Sex } \\
\hline Male & $89(60.14)$ & $64(53.33)$ & 1.252 & 0.263 \\
\hline Female & $59(39.86)$ & $56(46.67)$ & & \\
\hline \multicolumn{5}{|l|}{$\begin{array}{l}\text { Long-term } \\
\text { smoking }\end{array}$} \\
\hline Yes & $91(61.49)$ & $32(26.67)$ & 32.360 & $<0.001$ \\
\hline No & $57(38.51)$ & $88(73.33)$ & & \\
\hline \multicolumn{5}{|l|}{$\begin{array}{l}\text { Long-term } \\
\text { drinking }\end{array}$} \\
\hline Yes & $35(23.65)$ & $49(40.83)$ & 9.094 & 0.003 \\
\hline No & $113(76.35)$ & $71(59.17)$ & & \\
\hline \multicolumn{5}{|l|}{ Hypertension } \\
\hline Yes & $87(58.78)$ & $23(19.17)$ & 42.980 & $<0.001$ \\
\hline No & $61(41.22)$ & $97(80.83)$ & & \\
\hline \multicolumn{5}{|l|}{ Diabetes } \\
\hline Yes & $43(29.05)$ & $18(15.00)$ & 7.445 & 0.006 \\
\hline No & $105(70.95)$ & $102(85.00)$ & & \\
\hline \multicolumn{5}{|c|}{ Hyperlipidemia } \\
\hline Yes & $59(39.86)$ & $27(22.50)$ & 9.170 & 0.003 \\
\hline No & $89(60.14)$ & $93(77.50)$ & & \\
\hline \multicolumn{5}{|l|}{ BMI } \\
\hline$<18.5$ & $39(26.35)$ & $23(19.17)$ & 9.197 & 0.010 \\
\hline $18.50-24.9$ & $47(31.76)$ & $60(50.00)$ & & \\
\hline$>25.0$ & $62(41.89)$ & $37(30.83)$ & & \\
\hline
\end{tabular}

Comparison of Hcy and blood lipid levels in serum in different types of $C H D$. The study group was divided into angina pectoris, acute myocardial infarction and old myocardial infarction group according to the clinical manifestations of CHD patients. The difference was not statistically significant in the HDL-C level in patients among the three groups $(\mathrm{P}>0.05)$, but the differences were statistically significant in the levels of Hcy, TC, TG and LDL-C in serum of patients among the three groups $(\mathrm{P}<0.05)$. The Hcy level in acute and old myocardial infarction group was higher than that in angina pectoris group, and in old myocardial infarction group was lower than in acute myocardial infarction group $(\mathrm{P}<0.05)$; the TC level in angina pectoris group was higher than that in acute and old myocardial infarction group, and in acute myocardial infarction group was higher than in old myocardial infarction group $(\mathrm{P}<0.05)$; the TG level in acute and old myocardial infarction group was lower than that in angina pectoris group, and in old myocardial infarction group was lower than in acute myocardial infarction group $(\mathrm{P}<0.05)$; the LDL-C level in acute and old myocardial infarction group was lower than that in angina pectoris group, and in old myocardial infarction group was lower than in acute 
Table II. Comparison of Hcy and blood lipid levels in serum between the study and control group.

\begin{tabular}{lcccccc}
\hline Groups & $\mathrm{n}$ & Hcy $(\mu \mathrm{mol} / \mathrm{l})$ & $\mathrm{TC}(\mathrm{mmol} / \mathrm{l})$ & $\mathrm{TG}(\mathrm{mmol} / \mathrm{l})$ & HDL-C $(\mathrm{mmol} / \mathrm{l})$ & $\mathrm{LDL}-\mathrm{C}(\mathrm{mmol} / \mathrm{l})$ \\
\hline Study group & 148 & $21.06 \pm 5.28$ & $4.64 \pm 1.06$ & $1.96 \pm 0.33$ & $0.85 \pm 0.13$ & $2.29 \pm 0.57$ \\
Control group & 120 & $12.03 \pm 3.95$ & $4.21 \pm 0.62$ & $1.18 \pm 0.27$ & $1.41 \pm 0.26$ & $2.01 \pm 0.50$ \\
$\mathrm{t}$ & & 15.540 & 3.931 & 20.840 & 22.910 & 4.223 \\
P-value & $<0.001$ & $<0.001$ & $<0.001$ & $<0.001$ & $<0.001$ \\
\hline
\end{tabular}

Hcy, homocysteine; TC, total cholesterol; TG, triglyceride; HDL-C, high-density lipoprotein cholesterol; LDL-C, low-density lipoprotein cholesterol.

A
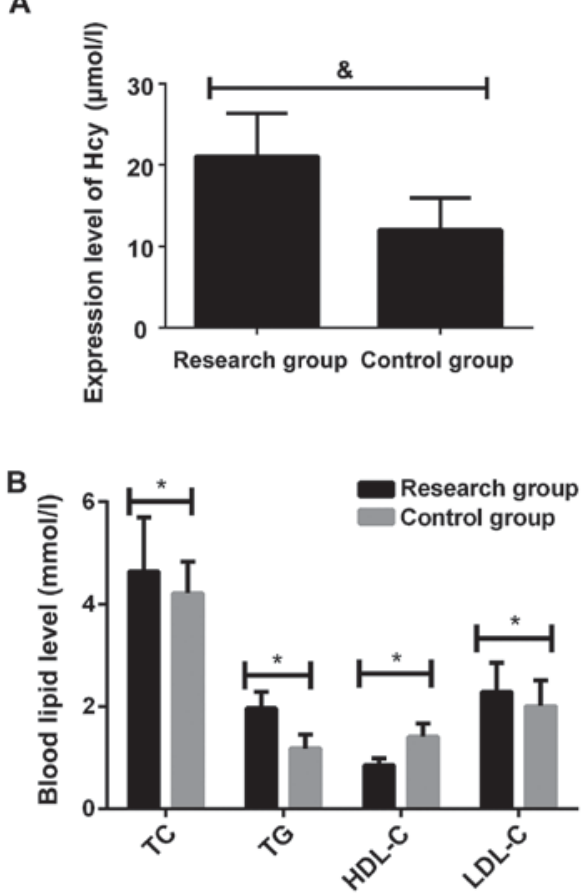

Figure 1. Comparison of Hcy and blood lipid levels in serum between the study and control group. (A) The results of enzyme-linked immunosorbent assay showed that the Hcy level in patients in the study group was significantly higher than that in control group $\left({ }^{\circledR} \mathrm{P}<0.001\right)$. (B) TC was determined using enzyme method, TG using glycerol phosphate oxidase method and HDL-C and LDL-C using selective inhibition method. The results showed that the levels of Hcy, TC, TG and LDL-C in patients in the study group were significantly higher than that in control group, while the HDL-C level was significantly lower than that in control group $(" \mathrm{P}<0.001)$. Hcy, homocysteine; TC, total cholesterol; TG, triglyceride; HDL-C, high-density lipoprotein cholesterol; LDL-C, low-density lipoprotein cholesterol.

myocardial infarction group $(\mathrm{P}<0.05)$. Specific information is shown in Fig. 2 and Table III.

Analysis of risk factors for CHD. BMI>25 was considered as obesity, and logistic regression analysis was performed on the factors with statistically significant difference used as independent variables. The results of univariate analysis showed that CHD was associated with Hcy, hyperlipidemia, smoking, hypertension and diabetes $(\mathrm{P}<0.05)$, but had no relationship with drinking and obesity $(\mathrm{P}>0.05)$. The results of multivariate analysis showed that Hcy, hyperlipidemia, hypertension and diabetes were independent risk factors for CHD, and the

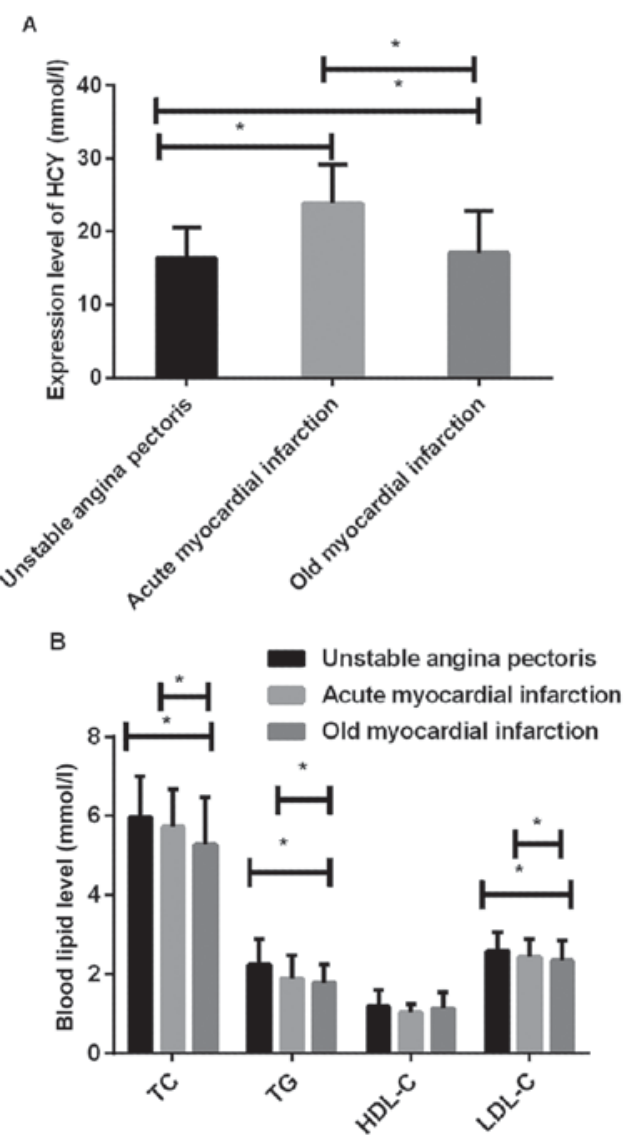

Figure 2. Comparison of Hcy and blood lipid levels in serum in different types of CHD. (A) The results of enzyme-linked immunosorbent assay showed that there was a significant difference in the Hcy level in patients among angina pectoris, acute myocardial infarction and old myocardial infarction group $\left({ }^{*} \mathrm{P}<0.05\right)$. (B) TC was determined using enzyme method, TG using glycerol phosphate oxidase method and HDL-C and LDL-C using selective inhibition method. The results showed that there was no significant difference in the HDL-C level in patients among angina pectoris, acute myocardial infarction and old myocardial infarction group $(\mathrm{P}>0.05)$. There was a significant difference in the levels of Hcy, TC, TG and LDL-C in patients among the three groups $(\mathrm{P}<0.05)$; the differences were statistically significant in the levels of Hcy, TC, TG and LDL-C between acute and old myocardial infarction and angina pectoris group $(\mathrm{P}<0.05)$; the differences were statistically significant in the levels of Hcy, TC, TG and LDL-C between old and acute myocardial infarction group $\left({ }^{*} \mathrm{P}<0.05\right)$. Hcy, homocysteine; $\mathrm{CHD}$, coronary heart disease; TC, total cholesterol; TG, triglyceride; HDL-C, high-density lipoprotein cholesterol; LDL-C, low-density lipoprotein cholesterol.

difference was statistically significant $(\mathrm{P}<0.05)$. Specific information is shown in Tables IV and V. 
Table III. Comparison of Hcy and blood lipid levels in serum in different types of CHD.

\begin{tabular}{lcccccc}
\hline Diseases & $\mathrm{n}$ & $\mathrm{Hcy}(\mu \mathrm{mol} / \mathrm{l})$ & $\mathrm{TC}(\mathrm{mmol} / \mathrm{l})$ & $\mathrm{TG}(\mathrm{mmol} / \mathrm{l})$ & HDL-C (mmol/l) & LDL-C (mmol/l) \\
\hline Angina pectoris & 67 & $16.42 \pm 4.16$ & $5.97 \pm 1.02$ & $2.24 \pm 0.65$ & $1.18 \pm 0.42$ & $2.59 \pm 0.47$ \\
Acute myocardial infarction & 49 & $23.81 \pm 5.37^{\mathrm{b}}$ & $5.72 \pm 0.95^{\mathrm{b}}$ & $1.89 \pm 0.58^{\mathrm{b}}$ & $1.03 \pm 0.21$ & $2.42 \pm 0.46^{\mathrm{b}}$ \\
Old myocardial infarction & 32 & $17.13 \pm 5.64^{\mathrm{a}, \mathrm{b}}$ & $5.26 \pm 1.21^{\mathrm{a}, \mathrm{b}}$ & $1.77 \pm 0.47^{\mathrm{a}, \mathrm{b}}$ & $1.13 \pm 0.41$ & $2.33 \pm 0.52^{\mathrm{a}, \mathrm{b}}$ \\
F-value & & 34.910 & 5.036 & 8.649 & 2.451 & 3.735 \\
P-value & & $<0.001$ & 0.008 & $<0.001$ & 0.090 & 0.026 \\
\hline
\end{tabular}

${ }^{\text {ap }}<0.05$, compared to acute myocardial infarction group; ${ }^{\text {}} \mathrm{P}<0.05$, compared to angina pectoris group. Hcy, homocysteine; CHD, coronary heart disease; TC, total cholesterol; TG, triglyceride; HDL-C, high-density lipoprotein cholesterol; LDL-C, low-density lipoprotein cholesterol.

Table IV. Univariate analysis of risk factors for CHD.

\begin{tabular}{lccc}
\hline & OR & $95 \%$ CI & P-value \\
\hline Smoking & 1.463 & $1.064-2.013$ & 0.031 \\
Drinking & 1.389 & $1.042-1.852$ & 0.076 \\
Hypertension & 0.871 & $0.672-1.130$ & 0.035 \\
Diabetes & 0.457 & $0.261-0.801$ & 0.029 \\
Obesity & 0.192 & $0.056-0.661$ & 0.054 \\
Hyperlipidemia & 0.893 & $0.772-1.108$ & 0.027 \\
Hcy & 1.814 & $0.925-3.558$ & 0.002 \\
\hline
\end{tabular}

CHD, coronary heart disease; Hcy, homocysteine.

Table V. Multivariate analysis of risk factors for CHD.

\begin{tabular}{lccc}
\hline & OR & $95 \%$ CI & P-value \\
\hline Smoking & 1.356 & $1.064-2.013$ & 0.064 \\
Hypertension & 0.843 & $0.776-1.130$ & 0.028 \\
Diabetes & 0.352 & $0.261-0.504$ & 0.031 \\
Hyperlipidemia & 0.759 & $0.634-1.034$ & 0.023 \\
Hcy & 1.736 & $0.925-3.687$ & 0.022 \\
\hline
\end{tabular}

CHD, coronary heart disease; Hcy, homocysteine.

\section{Discussion}

CHD is more common and even fatal in middle-aged and elderly people, the main pathological manifestation of which is cardiac insufficiency. Cardiac insufficiency is due to the coronary atherosclerotic plaque causing stenosis and blockage of arteries, thus inducing other clinical manifestations (12). A large number of previous reports have shown that the cholesterol level is associated with heart disease. However, in recent years, studies have shown that Hcy is an independent risk factor for CHD $(13,14)$. The specific reason is not clear why Hcy and blood lipid levels in serum are associated with CHD risk, but it has proved that CHD risk increases with the increase of Hcy and blood lipid levels in serum (15).

This study compared Hcy and blood lipid levels in serum between the study and control group. The HDL-C level in the study group was significantly lower than that in control group, while the levels of Hcy, TC, TG and LDL-C were significantly higher than that in control group, and the differences were statistically significant $(\mathrm{P}<0.001)$. The study of Granberry et al $(16)$ was consistent with our results that HDL-C had the effect of preventing atherosclerosis. Generally, the decrease of HDL-C level in CHD patients may be the result of the decrease of HDL-C content contributing to lipid deposition in the inner wall of the vessel, leading to the occurrence of CHD $(17,18)$. The difference was not statistically significant in the HDL-C level in patients among angina pectoris, acute myocardial infarction and old myocardial infarction group $(\mathrm{P}>0.05)$, but the differences were statistically significant in the levels of Hcy, TC, TG and LDL-C in serum of patients among angina pectoris, acute myocardial infarction and old myocardial infarction group $(\mathrm{P}<0.05)$. The differences were statistically significant in the levels of Hcy, TC, TG and LDL-C between acute and old myocardial infarction and angina pectoris group $(\mathrm{P}<0.05)$, and the differences were statistically significant in the levels of Hcy, TC, TG and LDL-C between old and acute myocardial infarction group $(\mathrm{P}<0.05)$. The study of Christensen et al (19) was consistent with our results, among which the Hcy level in patients in acute myocardial infarction group was significantly higher than that in the other two groups, suggesting that the cause of myocardial infarction in patients may be the increase of Hcy contributing to occurrence of thrombosis (20).

Analysis of risk factors for the disease was performed using logistic regression. The results of univariate analysis showed that CHD was associated with Hcy, hyperlipidemia, smoking, hypertension and diabetes $(\mathrm{P}<0.05)$, but had no relationship with drinking and obesity $(\mathrm{P}>0.05)$. The results of multivariate analysis showed that Hcy, hyperlipidemia, hypertension and diabetes were independent risk factors for CHD, and the difference was statistically significant $(\mathrm{P}<0.05)$. Our results are very similar to that of Menezes et al (21). According to statistics, smoking is associated with the death of CHD and is a significant risk factor for early onset of CHD, and especially the younger the smoker is, the greater the risk is (22). This is because long-term smoking speeds up the oxidation of LDL-C, resulting in decreased antioxidant capacity and dysfunction of blood vessel endothelium (23). Studies have shown that the incidence of CHD in hypertensive patients with high diastolic blood pressure is 5 times higher than that in patients with low diastolic blood pressure. About $70 \%$ of patients with type II diabetes die of CHD, the most important 
risk factor is dyslipidemia. In addition to some uncontrollable risk factors such as sex, age and genetic factors, people should actively control and cooperate to improve these variable risk factors $(24,25)$.

In conclusion, the Hcy level increases and the HDL-C level decreases in serum of CHD patients. Hcy, hyperlipidemia, hypertension and diabetes are independent risk factors for $\mathrm{CHD}$, which can provide reference for the assessment of the disease and for the early prevention and treatment.

\section{Acknowledgements}

Not applicable.

\section{Funding}

No funding was received.

\section{Availability of data and materials}

The datasets used and/or analyzed during the present study are available from the corresponding author on reasonable request.

\section{Authors' contributions}

WL drafted the manuscript. WL and TW were mainly devoted to the determination of Hcy and blood lipid levels in serum. PS and YZ was responsible for the statistical analysis. All authors read and approved the final manuscript.

\section{Ethics approval and consent to participate}

The study was approved by the Ethics Committee of Weifang People's Hospital (Weifang, China). Signed informed consents were obtained from the patients or the guardians.

\section{Patient consent for publication}

Not applicable.

\section{Competing interests}

The authors declare that they have no competing interests.

\section{References}

1. Zhong Y, Wang N, Xu H, Hou X, Xu P and Zhou Z: Ischemia-modified albumin in stable coronary atherosclerotic heart disease: Clinical diagnosis and risk stratification. Coron Artery Dis 23: 538-541, 2012.

2. Su Z, Zhang S, Hou Y, Zhang L, Liao L, Xia Q, Xiao C, Meng H and Yan Y: A preliminary study of single nucleotide polymorphisms of lipoprotein lipase gene in coronary atherosclerotic heart disease. Zhonghua Yi Xue Yi Chuan Xue Za Zhi 17: $157-160,2000$

3. Lyu Y, Jiang X and Dai W: The roles of a novel inflammatory neopterin in subjects with coronary atherosclerotic heart disease. Int Immunopharmacol 24: 169-172, 2015.

4. Ozkan Y, Ozkan E and Simşek B: Plasma total homocysteine and cysteine levels as cardiovascular risk factors in coronary heart disease. Int J Cardiol 82: 269-277, 2002.

5. Mayer O Jr, Simon J, Holubec L, Pikner R, Vobrubová I and Trefil L: Fibrate treatment and prevalence risk of mild hyperhomocysteinaemia in clinical coronary heart disease patients. Eur J Cardiovasc Prev Rehabil 11: 244-249, 2004.
6. Naureen A, Munazza B, Shaheen R, Khan SA and Fatima F: Serum homocysteine as a risk factor for coronary heart disease. $\mathrm{J}$ Ayub Med Coll Abbottabad 24: 59-62, 2012.

7. Wang P, Liu J, Lv X, Liu Y, Zhao Y and Guo W: A naphthalimide-based glyoxal hydrazone for selective fluorescence turn-on sensing of Cys and Hcy. Org Lett 14: 520-523, 2012.

8. Nabipour I, Vahdat K, Jafari SM, Amiri M, Shafeiae E, Riazi A, Amini AL, Pazoki R and Sanjdideh Z: Correlation of hyperhomocysteinaemia and Chlamydia pneumoniae IgG seropositivity with coronary artery disease in a general population. Heart Lung Circ 16: 416-422, 2007.

9. Sainani GS and Sainani R: Homocysteine and its role in the pathogenesis of atherosclerotic vascular disease. J Assoc Physicians India 50 (Suppl): 16-23, 2002.

10. Zhang HJ, Han P, Sun SY, Wang LY, Yan B, Zhang JH, Zhang W, Yang SY and Li XJ: Attenuated associations between increasing BMI and unfavorable lipid profiles in Chinese Buddhist vegetarians. Asia Pac J Clin Nutr 22: 249-256, 2013.

11. Sierakowska-Fijalek A, Kaczmarek P, Pokoca L, Smorag I, Wosik-Erenbek M and Baj Z: Homocystein serum levels and lipid parameters in children with atherosclerosis risk factors. Pol Merkur Lekarski 22: 146-149, 2007 (In Polish).

12. Wenger NK, Shaw LJ and Vaccarino V: Coronary heart disease in women: Update 2008. Clin Pharmacol Ther 83: 37-51, 2008.

13. Aronow WS: Association between plasma homocysteine and vascular atherosclerotic disease in older persons. Prev Cardiol 3: 89-91, 2000.

14. Doncheva N, Penkov A, Velcheva A, Boev M, Popov B and Niagolov Y: Study of homocysteine concentration in coronary heart disease patients and comparison of two determination methods. Ann Nutr Metab 51: 82-87, 2007.

15. Isaacs A, Willems SM, Bos D, Dehghan A, Hofman A, Ikram MA, Uitterlinden AG, Oostra BA, Franco OH, Witteman JC, et al: Risk scores of common genetic variants for lipid levels influence atherosclerosis and incident coronary heart disease. Arterioscler Thromb Vasc Biol 33: 2233-2239, 2013.

16. Granberry MC, Smith ES III and Talley JD: Raising HDL-C to prevent atherosclerotic events. J Ark Med Soc 96: 378-379, 2000.

17. Asztalos BF, Roheim PS, Milani RL, Lefevre M, McNamara JR, Horvath KV and Schaefer EJ: Distribution of ApoA-I-containing HDL subpopulations in patients with coronary heart disease. Arterioscler Thromb Vasc Biol 20: 2670-2676, 2000.

18. Paavola T, Kangas-Kontio T, Salonurmi T, Kuusisto S, Huusko T, Savolainen MJ and Kakko S: Plasma levels of antibodies against oxidized LDL are inherited but not associated with HDL-cholesterol level in families with early coronary heart disease. Atherosclerosis 224: 123-128, 2012.

19. Christensen B, Landaas S, Stensvold I, Djurovic S, Retterstøl L, Ringstad J, Berg K and Thelle DS: Whole blood folate, homocysteine in serum, and risk of first acute myocardial infarction. Atherosclerosis 147: 317-326, 1999.

20. Huang T, Yuan G, Zhang Z, Zou Z and Li D: Cardiovascular pathogenesis in hyperhomocysteinemia. Asia Pac J Clin Nutr 17: 8-16, 2008.

21. Menezes AR, Lavie CJ, Milani RV, Arena RA and Church TS: Cardiac rehabilitation and exercise therapy in the elderly: Should we invest in the aged? J Geriatr Cardiol 9: 68-75, 2012.

22. Bruce IN: 'Not only...but also': Factors that contribute to accelerated atherosclerosis and premature coronary heart disease in systemic lupus erythematosus. Rheumatology (Oxford) 44: 1492-1502, 2005.

23. Lynch J, Davey Smith G, Harper S and Bainbridge K: Explaining the social gradient in coronary heart disease: Comparing relative and absolute risk approaches. J Epidemiol Community Health 60: 436-441, 2006.

24. Marijon E, Trinquart L, Jani D, Jourdier H, Garbarz E, Mocumbi AO, Celermajer DS and Ferreira B: Coronary heart disease and associated risk factors in sub-Saharan Africans. J Hum Hypertens 21: 411-414, 2007.

25. Maas AH, van der Schouw YT, Beijerinck D, Deurenberg JJ, Mali WP and van der Graaf Y: Arterial calcifications seen on mammograms: Cardiovascular risk factors, pregnancy, and lactation. Radiology 240: 33-38, 2006.

This work is licensed under a Creative Commons Attribution-NonCommercial-NoDerivatives 4.0 International (CC BY-NC-ND 4.0) License. 